\title{
Interstitial lung diseases in infants and
} children

\author{
A. Clement* and E. Eber ${ }^{\#}$
}

ABSTRACT: Interstitial lung disease (ILD) in infants and children represents a heterogeneous group of respiratory disorders that are mostly chronic and associated with high morbidity and mortality.

Typical features of ILD include dyspnoea, the presence of diffuse infiltrates on chest radiographs and abnormal pulmonary function tests with evidence of a restrictive ventilatory defect (in older children) and/or impaired gas exchange.

ILD is difficult to diagnose, as no classification scheme is entirely satisfactory. Recently, it has been proposed to categorise paediatric ILD as either primary pulmonary disorders or systemic disorders with pulmonary involvement. However, this classification leaves the difficult problem of which clinical conditions should be included or excluded in infants and children.

The present article reviews interstitial lung disease in infants and children, placing emphasis on current concepts of pathophysiological mechanisms and approaches to treatment.

KEYWORDS: Alveolar epithelium, growth factors, interstitial lung diseases in children, pathogenesis of fibrosing process, prognosis, therapy

I nterstitial lung disease (ILD) in children comprises a broader spectrum of disorders than in adults $[1,2]$. This is certainly linked to the fact that the disease occurs in the context of lung growth at the various stages of alveolar development and maturation, with each of these stages being regulated by specific cascades of events [3]. In children, ILD is most frequently diagnosed in the first year of life with a predominance of paediatric entities, such as pulmonary interstitial glycogenosis, neuroendocrine cell hyperplasia of infancy and genetic disorders of surfactant metabolism [1, 4]. In older children, the pathogenesis of ILD shares similarities with the processes observed in adults, and the pathological changes are characterised by derangement of the alveolar septum with the presence of focal zones of fibroblast proliferation called fibroblastic foci. These patchy fibrotic lesions appear to occur at sites of recent alveolar injury, and their number seems to correlate with worsening lung function and poor prognosis. The data on pathophysiological mechanisms as presented in the current review article is mostly derived from studies in adult patients or animals.

\section{CURRENT CONCEPTS OF THE} PATHOPHYSIOLOGICAL MECHANISMS

\section{Epithelial-mesenchymal cross-talk as} pathogenic pathway

Underlying mechanisms

For a long time, the accepted view has emphasised the key role of a persistent alveolitis that injures the lung and modulates fibrogenesis, regardless of initiating agents [5]. The development of a chronic inflammatory response was thought to perpetuate the recruitment of inflammatory and immunoregulatory cells into the interstitium, alveolar walls and peri-alveolar tissues, progressively leading to a thickened alveolar wall with extensive fibrosis and loss of the alveolar gas exchange function. However, analysis of better-defined cases of ILD in adult patients has revealed that prominent inflammation was mainly present at an early stage and was mostly documented in pathological situations that often did not progress to end-stage fibrosis, such as hypersensitivity pneumonitis. By contrast, in diseases with an extensive fibrotic pattern, the inflammatory component was usually mild. Reports from a number of studies have shown that clinical measurements of

\section{AFFILIATIONS}

*Paediatric Pulmonary Dept, AP-HP, Hôpital Trousseau, Université Pierre et Marie Curie-Paris 6, Paris, France.

\#Respiratory and Allergic Disease Division, Paediatric Dept, Medical University of Graz, Graz, Austria.

\section{CORRESPONDENCE}

E. Eber

Respiratory and Allergic Disease Division

Paediatric Dept

Medical University of Graz

Auenbruggerplatz 30

A-8036 Graz

Austria

Fax: 433163854621

E-mail: ernst.eber@meduni-graz.at

Received:

January 122007

Accepted after revision:

November 172007

STATEMENT OF INTEREST

None declared.
European Respiratory Journal Print ISSN 0903-1936

Online ISSN 1399-3003 
inflammation did not correlate with disease stage and outcome [5]. In addition, if ILD was mainly a disease of an excessive inflammatory process with subsequent remodelling of the lung by fibrosis, efficacy of anti-inflammatory therapy should be well established by now. However, in many cases of ILD in adult patients, glucocorticosteroids have failed to improve disease outcome, even when administered at high doses and in combination with potent immunosuppressive drugs. Some beneficial effects were only reported if glucocorticosteroid therapy was begun at an early stage during the inflammatory phase [6]. In addition, overexpression of pro-inflammatory mediators, such as interleukin (IL)-8, in rodent lung was not found to be associated with marked progressive chronic fibrosis. This does not mean that inflammation with alveolitis is not present at an early stage of the disease. Indeed, following any form of injury, an inflammatory response is initiated that stimulates proliferation of mesenchymal and epithelial cells and matrix production [6-8]. This response is usually followed by apoptosis of repair cells and matrix mobilisation with fibrolysis to ensure a proper healing. In the situation of ILD, the pathological process does not appear to be linked to an ongoing and chronic inflammatory response, but rather to a continued matrix production with a reduced matrix mobilisation and to an altered apoptosis of the alveolar cells [9-11]. Consequently, evidence has progressively emerged that inflammation may not be the prominent factor for development of the fibrotic response.

Recently, much interest has been focused on the injury of the alveolar surface with the marked disruption in the integrity of the epithelium during the course of the disease [12, 13]. ILD develops following epithelial damage; this induces the accumulation and activation of immuno-inflammatory cells, with subsequent migration and proliferation of fibroblasts and deposition of extracellular matrix. Progressively, foci of fibroblasts/myofibroblasts within the alveolar interstitium can be individualised on lung tissue biopsies of patients. This pathological pattern is now thought to be the result of abnormal repair of the lung. Under physiological conditions, the alveolar epithelium responds to injury by an adequate process of wound healing to restore lung surface integrity. A key step in this process is the capacity of alveolar epithelial type 2 cells to initiate re-epithelialisation rapidly. Indeed, these cells represent the stem cells of the alveolar epithelium due to their ability to proliferate and to undergo transition into the terminally differentiated type 1 cells. In situations of extensive damage to the lung surface, there will be a delay in the initiation and progression of the re-epithelialisation process $[14,15]$. Consequently, prolonged denudation of the basement membrane may contribute to altered interactions and crosstalk between alveolar epithelial cells and mesenchymal cells, resulting in profound modifications of cell functions with imbalanced production of polypeptide mediators, including cytokines, growth factors, oxidants and proteases $[8,16]$. The local population of fibroblasts and myofibroblasts may progressively increase due to stimulation of proliferation by local mitogenic factors and reduction of apoptosis. This may lead to progressive aberrant tissue remodelling by disorganisation of extracellular matrix component deposition, including fibrillar collagen, elastic fibres, fibronectin and proteoglycans. In addition, the abnormal lung architecture observed in pulmonary fibrosis appears to be associated with the formation of new blood vessels. This process requires the secretion of angiogenic molecules to promote endothelial cell migration and neovascularisation (fig. 1).

\section{Major cellular actors}

The distinctive feature in a fibrotic lung, i.e. a marked disruption of the alveolar epithelium, probably results from a dysfunctional epithelial repair process involving altered differentiation leading to phenotype changes and abnormal proliferative response. This contributes to the failure to replace damaged type 1 cells and to abnormalities in pulmonary surfactant production with alveolar collapse. Recent reports suggest the possibility that epithelial cells, when detached from their basement membrane, may enter into epithelialmesenchymal transition with the appearance of reactive and elongated alveolar epithelial cells [8]. The presence of hyperplastic epithelial areas in the fibrotic lung together with areas of epithelial cell loss is the result of an inadequate proliferative response. The reasons for epithelial cell loss and inappropriate re-epithelialisation remain unclear. Recently, much interest has been focused on epithelial cell apoptosis and cell removal $[8,17]$. A common feature of cells undergoing apoptosis is cell surface expression of phosphatidylserine, a molecule normally present on the inner part of the plasma membrane. Recognition of phosphatidylserine by specific receptors on neighbouring cells is associated with the production of several anti-inflammatory molecules, including transforming growth factor (TGF)- $\beta$ [18-21]. TGF- $\beta$ is thought to play an important role by allowing apoptotic cells to be cleared with minimal local reaction. In normal situations, the progressive diminution of apoptotic cells results in a decreasing supply of TGF- $\beta$. TGF- $\beta$ has been shown to be overexpressed in fibrotic lung disorders. One explanation for the welldocumented increased production of TGF- $\beta$ involves upregulation of the phosphatidylserine receptors through an altered balance of the release of proteases and anti-proteases. A consequence would be the perpetuation of a vicious cycle with TGF- $\beta$ promoting epithelial cell apoptosis, which in turn increases the local production of TGF- $\beta$. Downstream events

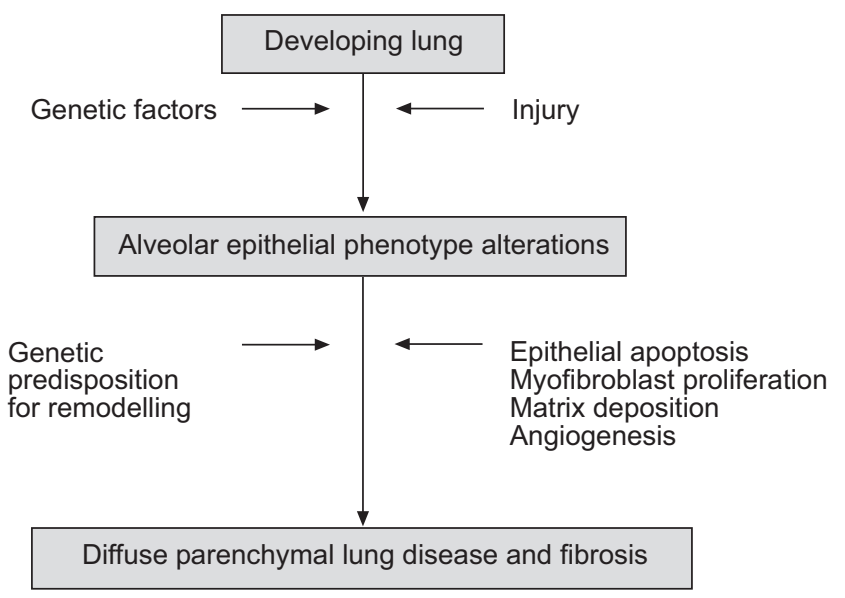

FIGURE 1. Schematic representation of the proposed mechanism of diffuse alveolar damage and fibrosis in the developing lung. 
linked to upregulation of TGF- $\beta$ include modifications in the expression of various components of the cell cycle machinery, mainly the cyclin-dependent kinases (CDK) system which plays an essential role in ensuring proper cell cycle progression. Recently, much work has been focused on the protein p21cip1, a member of the CDK inhibitor family. This protein promotes cell cycle arrest to apoptosis in cases of cellular DNA damage. Interestingly, upregulation of p21cip1 has been reported in the lung tissues of patients with pulmonary fibrosis, primarily in hyperplastic alveolar epithelial cells. This increased expression of p21cip1 may favour the process of epithelial cell apoptosis [3, 21].

Another important cellular factor in the altered re-epithelialisation process is the fibroblast [22]. Histopathological analyses of fibrotic lungs have repeatedly documented the presence of fibroblastic/myofibroblastic foci. In response to injury, alveolar epithelial cells and inflammatory cells release and activate a number of cytokines and growth factors involved in fibroblast migration, proliferation and change to myofibroblasts, leading to accumulation and remodelling of the extracellular matrix. These factors include TGF- $\beta$, insulin-like growth factor (IGF)-1, platelet-derived growth factor, connective tissue growth factor, keratinocyte growth factor, hepatocyte growth factor and proinflammatory cytokines, such as tumour necrosis factor (TNF)$\alpha$. It is likely that the formation of myofibroblasts is due to a series of events, with progressive transformation of fibroblasts: initially a migratory phenotype, then a proliferative phenotype and, finally, a myofibroblast contractile profibrotic phenotype. Myofibroblasts certainly participate in the remodelling fibrotic process through several mechanisms, which include production of matrix metalloproteinases. The disappearance of myofibroblasts represents progression towards resolution of the pathological process and subsequent healing. Emergence of the fibroblast phenotypes is regulated by a variety of factors. Among them is TGF- $\beta$ with its capacity to promote myofibroblast survival and persistence. Interestingly, one paradox of fibrosis is that the altered repair process is associated with decreased apoptosis in myofibroblasts and increased apoptosis in epithelial cells. New insights into the mechanisms of myofibroblast emergence include the relationship between loss of telomerase expression and induction of $\alpha$-smooth muscle actin expression in the transition to the myofibroblast phenotype. A recent development also questions the origin of the cells in the fibrotic foci. For a long time, it has been claimed that fibroblasts derive from pre-existing resident lung fibroblasts under the influence of mediators produced by local cells. There are now reports suggesting that myofibroblasts may be derived from nonresident, circulating cells called fibrocytes $[22,23]$. These fibrocytes may be recruited to the lung in cases of fibrotic disorders and may then differentiate into various fibroblast phenotypes. In addition, recent data support the hypothesis that alveolar epithelial cells may serve as a source of myofibroblasts through epithelial-mesenchymal transition. This is not restricted to the lung. Indeed, studies of renal fibrosis suggest that one-third of the fibroblasts at sites of injury arise from epithelial cells [23].

\section{Key molecular factors influencing the alveolar repair process}

A cascade of mediators produced locally by epithelial, mesenchymal and inflammatory cells plays a critical role in the progression of the fibrotic changes. Among them are TNF- $\alpha$ and the T-helper cell (Th) type 2 cytokines [24, 25]. The inflammatory response documented in patients with pulmonary fibrosis has been reported to resemble a Th2 immune response with eosinophils and increased levels of Th2 cytokines [12]. These clinical data are sustained by experimental studies indicating that when mice are exposed to various types of lung injury, progression towards development of pulmonary fibrosis is mainly observed in animals with a predominant Th2 response. While the mechanisms leading to the shift in the acquired immune response to a cytokine phenotype characterised by high production of IL-4 and -13 remain unclear, the influence of the Th2 response in the fibrotic process is starting to become clear. Mice overexpressing IL-13 have been reported to develop eosinophilic inflammation and epithelial airway fibrosis [8,9]. In addition, IL-13 can activate fibroblasts resulting in elevated collagen production, most probably through increased expression of the TGF- $\beta$ cascade. Consequently, targeting Th2 cytokines should certainly be considered as an anti-fibrotic approach. The use of immunomodulators, such as interferon (IFN)- $\gamma$, may represent a therapeutic strategy to switch the inflammatory response to a more Th1-like phenotype.

An important multifunctional cytokine acting at various stages of the disease is TGF- $\beta$. TGF- $\beta$ isoforms regulate various cell functions, including cell proliferation, cell differentiation, apoptosis, cell adhesion/motility and matrix production. In vivo studies have demonstrated increased TGF- $\beta$ gene expression and protein secretion in the lung of animals and humans with fibrotic disease [26]. The fact that pathological fibrosis is mediated by TGF- $\beta$ is supported by several findings, such as the following: 1) tissue damage increases TGF- $\beta$ production before the production of extracellular matrix increases; 2) TGF$\beta$ is a potent stimulator of the production and deposition of extracellular matrix; 3 ) TGF- $\beta$ induces fibrosis independently of tissue damage; and 4) inhibitors of TGF- $\beta$ receptor binding either reduce or abolish fibrosis. In addition, overexpression of active TGF- $\beta$ in rat lung was shown to result in severe interstitial fibrosis and emergence of cells with the myofibroblast phenotype. Recently, it has been reported that exposure of alveolar epithelial cells to TGF- $\beta$ resulted in increased expression of mesenchymal markers, including $\alpha$-smooth muscle actin, type 1 collagen and vimentin, and decreased expression of epithelial markers [23]. This effect occurred through the phosphorylation of Smad2 and was inhibited by Smad2 gene silencing [26].

\section{Pathogenesis of ILD in the context of lung growth}

The frequency of ILD and other fibrotic disorders is much lower in children than in adults. Certainly, some clinical situations have features unique to children but many of these diseases overlap their adult counterparts, with the primary event being injury to and damage of the alveolar epithelium. However, the overall outcome and prognosis of the diseases in children are thought to be less severe than in adult patients, and paediatric ILD is more responsive to therapeutic strategies than adult ILD [4]. These differences may be explained by the types of initial injuries, which may not be similar, due to changes in the host environment. Another explanation is the modification of the process of wound healing with age [27]. 
The process of healing involves the coordinated regulation of cell proliferation and migration and tissue remodelling, predominantly by polypeptide growth factors. The slowing of wound healing that occurs in the aged may be related to changes in the activity of these various regulatory factors. In a recent study on the role of ageing in the development of cardiac fibrosis in the rabbit, differences in the cascade of events leading to myocardial remodelling were observed: these were mainly the presence of more myofibroblasts synthesising collagen and the expression of high levels of TGF- $\beta$ in the old animals [28]. A study of skin wound healing in young and aged mice also showed age-dependent changes. Expression of all the fibroblast growth factors was diminished in aged mice, even in healthy skin [29]. In addition, the post-wound regulation of expression of these factors and of TGF- $\beta$ was less pronounced and slower than in the young mice [30]. These findings are in agreement with data observed in muscle that indicated significant alterations in the TGF- $\beta$ production with age. Indeed, muscle cells from older animals exhibited major alterations in the expression levels of many genes either directly or indirectly involved with TGF- $\beta$ signalling pathways. In ILD, an essential step for the restoration of alveolar integrity is the rapid re-epithelialisation of the altered surface, mainly through epithelial proliferation and migration processes, as previously discussed. These are dependent on a balanced availability of growth factors, with TGF- $\beta$ being an important component of this balance. In young patients, it can be suggested that the programmed production of mitogenic factors, together with a more adapted regulation of TGF- $\beta$ expression, may favour the process of re-epithelialisation and may help to counteract the altered secretion of mediators involved in migration and proliferation of fibroblasts, as well as in differentiation into myofibroblasts.

\section{Genetic factors}

Epidemiological studies have confirmed the occurrence of familial cases, with an estimated prevalence ranging 1.3-5.9 per million [31]. The familial form of ILD is probably transmitted as an autosomal dominant trait with reduced penetration. Among the recent genetic findings has been the identification of causal mutations in the surfactant protein (SP) genes, mainly in SP-B and -C genes [32-34]. SP-B and SP-C are hydrophobic proteins that interact with surfactant lipids to facilitate adsorption to the air-liquid interface. Defects in SP-B, when homozygous for the most common mutation (121ins2) are highly lethal in the first few months of life. Reported patients with partial SP-B deficiency were bearing mutations leading to either production of small amounts of SP-B or to pro-SP-B, which was not further processed to mature SP-B [34]. In these various conditions, the outcome is dependent on the amount of SP-B produced, as it is believed from animal models that a critical level of SP-B is required for physiological lung function. For SP-C, several mutations have been described, the first being a guanine $(\mathrm{G})$ to adenine $(\mathrm{A})$ substitution on the first base of intron 4 (IVS4+1G>A), causing skipping of exon 4 with the deletion of 37 amino acids. Abnormal expression of SP-C is associated with variable phenotypes. Patients with SP-C mutations can present with severe manifestations early in life or may develop symptoms in adulthood [33]. Recently, mutations in other genes involved in the surfactant metabolism have been reported. Among them are the mutations in the
ABCA3 gene [34]. ABCA3 is a member of the ATP binding cassette protein family. It is highly expressed in alveolar epithelial cells at the limiting membrane of lamellar bodies, and may play a role in lipid homeostasis of lamellar bodies. The phenotypes of ABCA3 need further evaluation.

In most of the ILD cases, no family history can be documented. In these situations, it is likely that multiple genetic factors may contribute to a modest effect on predisposition to the disease, in combination with appropriate environment. Recently, several case-control association studies have been reported [31]. Although many candidate genes can be proposed, only limited numbers have been evaluated and have confirmed associations. Among them are polymorphisms in TGF- $\beta 1$, TNF- $\alpha$, IL-1 cluster, chemokine-related genes, angiotensinconverting enzyme gene and IFN- $\gamma$ gene. These reports are from adult studies [31]. No information is currently available in the paediatric population.

\section{CURRENT AND NOVEL APPROACHES TO DIAGNOSIS AND TREATMENT \\ Diagnosis}

Recently, a European Respiratory Society (ERS) Task Force on chronic interstitial lung disease in immunocompetent children collected the largest series of children with ILD $(n=185)$ in the world literature to date, a cohort fairly representative of current practice across Europe [1].

The main conclusion of this ERS Task Force was that major variations exist between the various centres regarding disease classification and evaluation, and that standardisations are urgently needed. Consequently, Task Force members developed detailed recommendations for the diagnosis of chronic ILD in children, including recommendations for clinical evaluation, laboratory tests, chest imaging, pulmonary function testing, bronchoalveolar lavage (BAL) studies, tissue biopsies and the histopathological approach; the interested reader is referred to the original article [1].

Paediatric ILD comprises a large spectrum of disorders. However, there is now compelling evidence that some of these disorders are observed more frequently in infants, while others are more specific to older children. Consequently, the stage of lung development and maturation should be taken into account in order to provide a more appropriate approach to the diagnosis of paediatric ILD. This implies new strategies in the discussion of the tests that need to be performed when the patients are referred to specialised centres. In infants, some unique forms of ILD should be considered first, with the use of appropriate tools. These disorders include neuroendocrine cell hyperplasia of infancy, pulmonary glycogenosis, genetic defects of surfactant function, and disorders of lung growth and development. In older patients, the list of differential diagnoses to work on is highly dependent on the history and clinical presentation. Primary pulmonary disorders can be suggested, such as hypersensitivity pneumonitis, pulmonary alveolar proteinosis, alveolar haemorrhage syndromes, druginduced lung disease, and pulmonary lymphatic and vascular disorders. In other situations, systemic disorders with pulmonary involvement will be discussed. These include Langerhans cell histiocytosis, sarcoidosis, lipid storage disease and connective tissue disease $[35,36]$. For these various 
diagnoses, both noninvasive and invasive tools are currently available. In the absence of a specific diagnosis, the term "idiopathic ILD" will be retained, based on compatible clinical, radiological and pathological findings.

\section{Treatment}

Even in adults, where chronic ILD is at least two orders of magnitude more common than in children [37], a major problem has been the lack of large, randomised clinical trials to guide treatment of many of these diseases [38]. Most current treatment regimens for children are not only based on experience gained in small numbers of patients within individual centres, but are also derived from information provided by studies in adult patients, although there is increasing evidence that expression and outcome of chronic ILD in children differ from adult ILD [39]. In general, these treatment strategies are unsatisfactory, and morbidity and mortality are still considerable.

In the series collected by the ERS Task Force on chronic ILD in children [1], the management was not standardised but varied from centre to centre, depending on both resources and expertise. In this retrospective analysis, it was not possible to relate the outcome to the type of ILD or the therapeutic strategy. Again, the interested reader is referred to the original article for detailed information on both currently employed treatment strategies and treatment recommendations [1].

As previously indicated, paediatric ILD comprises a heterogeneous group of predominantly rare disorders. These diseases not only present enormous diagnostic challenges but, in addition, their natural history is extremely variable [4, 40]. Thus, treatment clearly depends on the severity of the disease.

\section{General measures}

As in any child with chronic lung disease, supportive care includes administration of oxygen for chronic hypoxaemia, adequate nutrition, annual immunisation with influenza vaccine along with other routine immunisations against respiratory pathogens, aggressive treatment of intercurrent infections, strict avoidance of tobacco smoke and other air pollutants, and the selective use of bronchodilators. In addition, the treatment of associated gastro-oesophageal reflux, whether it is a primary or secondary phenomenon, may be important in the management of children with ILD.

\section{Pharmacological therapy}

A few children with very mild disease do not require any treatment and recover spontaneously. The clinical condition of infants with neuroendocrine hyperplasia of infancy, often being symptomatic and requiring oxygen for months to years, has been reported to gradually improve over time, possibly with no treatment [4]. In the majority of infants and children with ILD, however, treatment with immunosuppressive, antiinflammatory, or antifibrotic drugs is required for weeks, months or even years [1, 41, 42]. For the reasons mentioned previously, very little progress has been made regarding pharmacological treatment during recent decades. On the assumption that suppression of inflammation might be beneficial, corticosteroids are still the most commonly used drugs for a number of conditions, and a trial of corticosteroids for $\geqslant 6-8$ weeks should be considered in every child with idiopathic ILD. However, responses to steroids have been found to be highly variable in many types of ILD [4, 42]. Naturally, patients requiring long-term corticosteroid therapy are at risk of developing severe side-effects; among others there is the potential for long-term adverse effects on neuromotor and cognitive function, especially in very young infants [43]. Thus, the considerable morbidity and mortality associated with many forms of ILD has to be balanced against the treatment risk, and any medication has to be titrated to the lowest dose resulting in clinical stability.

The most commonly used treatment still consists of oral prednisolone [42]. However, as pulsed steroid therapy appears to be associated with fewer side-effects, today this therapeutic strategy is preferred by some authors, particularly in children with significant disease $[4,44,45]$. There is anecdotal evidence that high-dose intravenous methylprednisolone pulses may work even when other steroids fail; in addition, it may be speculated that downregulation of glucocorticoid receptors, which can be seen in daily treatment with steroids, can be avoided by pulse therapy [44, 46]. So far, however, there are several unresolved issues with corticosteroid therapy. These pertain to the following: 1) the doses and the frequency at which i.v. methylprednisolone pulses should be performed; 2) the criteria that should be used for discontinuation of the treatment; 3) the doses and the (dis)advantages of different oral prednisolone regimens; and 4) the potential usefulness of inhaled corticosteroids.

A number of alternative or steroid-sparing agents has been used with anecdotal success in children with ILD. Of these, the antimalarial agents chloroquine and hydroxychloroquine have probably been used most frequently [42, 47, 48]. Chloroquine treatment has been shown to be associated with irreversible retinopathy; in contrast, the risk of retinopathy among patients receiving appropriate doses of hydroxychloroquine is negligible, making hydroxychloroquine the preferred agent [49]. Some authors feel that, if predominant lung biopsy findings are desquamation and inflammation, steroids appear to be the preferable therapeutic approach. However, the concept that successful treatment with corticosteroids is related to the degree of inflammation has not been proven in children. In contrast, in patients with milder disease, particularly when (pre-)fibrotic changes prevail over desquamation and inflammation in lung biopsy specimens, hydroxychloroquine may result in a more favourable outcome [1]. However, similar to steroids, responses to chloroquine and hydroxychloroquine are highly variable in many types of ILD [41]. Patients with severe histological changes will probably require both agents.

When steroids and hydroxychloroquine are not successful, or when there is evidence of severe steroidal side-effects, other immunosuppressive or cytotoxic agents, such as azathioprine, cyclophosphamide, cyclosporine or methotrexate, may be used. There is some experience with these treatment options in children with autoimmune disorders, such as Wegener's granulomatosis, dermatomyositis and others [50, 51].

\section{Specific treatment strategies}

Patients with underlying systemic disorders require primary treatment for that disorder, e.g. chemotherapy for malignancy, 
or i.v. $\gamma$-globulin for hypogammaglobulinaemia. Obviously, specific therapeutic strategies are preferable to less specific treatment. These include anti-infective therapy for chronic respiratory infections (e.g. cytomegalovirus or Epstein-Barr virus infection) [52], IFN- $\alpha$ for pulmonary haemangiomatosis [53], and whole-lung lavage or granulocyte-macrophage colony-stimulating factor (GM-CSF) in idiopathic pulmonary alveolar proteinosis [54,55]. While original reports described subcutaneous administration of GM-CSF [55], more recently, patients with idiopathic pulmonary alveolar proteinosis were successfully treated with aerosolised GM-CSF, including one child $[56,57]$. In patients with hypersensitivity pneumonitis, avoidance of the causative environmental antigen should be attempted. Furthermore, in patients treated with corticosteroids or cytotoxic agents chronically, prophylaxis against Pneumocystis jirovecii with trimethoprim/sulfamethoxazole should be considered.

\section{Transplantation}

Lung or heart-lung transplantation may be offered as the ultimate therapy for end-stage ILD and for some lethal diseases, such as those caused by mutations in the surfactant protein $\mathrm{B}$ and $\mathrm{ABCA} 3$ genes [41]. In recent years, transplantation has been shown to be a viable option in children of all ages, even in young infants [58,59]. This treatment option, however, is clouded by the fact that a very high percentage of long-term survivors of lung transplantation will develop bronchiolitis obliterans, and bronchiolitis obliterans or its complications are the single most common cause of death in these patients [60]. In addition, there have been anecdotal reports on the recurrence of several ILDs in the recipient lung, including sarcoidosis, Langerhans cell histiocytosis, idiopathic pulmonary haemosiderosis and desquamative interstitial pneumonitis [38, 61, 62].

\section{Novel therapeutic approaches}

As conventional therapy with corticosteroids and cytotoxic agents is often ineffective, a number of novel therapeutic strategies based on increased knowledge of ILD pathogenesis are being developed [63]. These new approaches to treatment of ILD in particular involve substances directed against the action of cytokines, growth factors and oxidants. Several of these agents have shown promise at the clinical trial stage in adult patients with idiopathic pulmonary fibrosis (IPF). To date, encouraging results have been obtained with IFN- $\gamma$, a cytokine with diverse properties, including antifibrotic, antiinfective, antiproliferative (inhibition of the proliferation of lung fibroblasts in a dose-dependent manner), and immunomodulatory effects. In a preliminary study, 12 months of treatment with IFN- $\gamma$ in combination with prednisolone was associated with substantial improvement of pulmonary ventilation and gas exchange in patients with IPF who had shown no response to glucocorticoids alone [64]. Another placebocontrolled trial of IFN- $\gamma$ in patients with IPF over a 1-yr period could not demonstrate a significant effect on progression-free survival, pulmonary function or quality of life, although due to the size and the duration of the trial, a clinically significant survival benefit could not be ruled out [65]. Furthermore, a recent meta-analysis suggests that IFN- $\gamma$ therapy in patients with IPF is associated with reduced mortality [66]. Beneficial effects have also been reported for other antifibrotic agents, such as pirfenidone. In a prospective, open-label study in patients with advanced IPF, treatment with pirfenidone appeared to arrest further decline in lung function in the majority and improved oxygenation in a few patients [67]. A subsequent double-blind, randomised, placebo-controlled trial demonstrated improvement of lung function and prevention of acute exacerbations in pirfenidone-treated patients; however, the study was aborted in favour of pirfenidone treatment due to an increased number of acute exacerbations in the placebo group [68].

In patients with pulmonary sarcoidosis, another cytokinebased approach, i.e. inhibition of TNF- $\alpha$, holds promise for the future. Recently, patients with various manifestations of sarcoidosis, whose disease was refractory to other agents or in whom other agents were poorly tolerated, were treated with infliximab, a chimeric monoclonal antibody directed against TNF- $\alpha$. This retrospective study demonstrated objective evidence of improvement in all patients and concluded that infliximab treatment appears to be safe [69]. In addition, pentoxifylline has been shown to suppress TNF- $\alpha$ production and thus may be another treatment option for sarcoidosis, either by sparing or by replacing corticosteroids [70]. While this drug has been reported as successful in the treatment of active pulmonary sarcoidosis, its precise clinical value will have to be determined in further clinical trials [71]. Etanercept, which, by binding TNF- $\alpha$, blocks the interaction of TNF- $\alpha$ with its receptor, was reported to be frequently associated with early or late treatment failure in patients with progressive stage II or III pulmonary sarcoidosis [72]. Thalidomide, another cytokine modulator that blocks TNF- $\alpha$ release from alveolar macrophages, has been shown to be an effective form of treatment for chronic cutaneous sarcoidosis but does not appear to be as effective for pulmonary disease [73].

Obviously, none of the presented studies in IPF and sarcoidosis patients included infants and children; thus, it presently remains unclear whether some of the reported benefits with these novel approaches may also be achieved in the treatment of paediatric ILD given the marked differences in wound healing and scar formation and prognosis between infants/young children and adults.

In addition, there is evidence that proteinases are involved in the promotion of both the initial lung injury and the following fibroproliferative repair in fibrotic lung diseases [10]. Thus, antiproteolytic strategies might represent effective forms of treatment in these diseases, and this may have wide implications for the future treatment of children with ILD. With the potential to deliver specific inhibitors of inflammation or collagen biosynthesis directly to the lung via aerosols, the lung may be quite amenable to such novel therapies.

\section{Prognosis}

The prognosis for children with ILD is extremely variable. While some patients with the newly described entities cellular interstitial pneumonitis/pulmonary interstitial glycogenosis and persistent tachypnoea of infancy/neuroendocrine cell hyperplasia of infancy generally do well in the long term, many of those with genetic abnormalities of surfactant function and other types of ILD do poorly. Furthermore, some children with relatively severe fibrosis on biopsy may make good 
progress, whereas others with only mild desquamation may have a poor outcome. This might be due to variable severity of the diseases in different parts of the lung, despite biopsies being taken guided by high-resolution computed tomography (HRCT). In summary, no correlation between the response to treatment or the clinical outcome and the initial pattern of chest radiograph or HRCT scan changes or the histological findings in lung biopsy specimens has been demonstrated so far $[42,74]$. To date, there are only very limited data on the long-term outcome regarding lung function in children with ILD [42].

The overall mortality rate is around $15 \%$, with a very variable outcome reported for infants [42, 44, 74-76]. A severity-ofillness score using symptoms, oxygen saturation at rest and during exercise or sleep, and pulmonary hypertension was reported to be a useful means of predicting the probability of survival [75]. The majority of children recover and most of them can lead normal lives [42]. Only a few patients are left with significant sequelae, such as limited exercise tolerance or the need for long-term oxygen therapy.

\section{CONCLUSION}

Ongoing basic research will provide new insights into the molecular basis of interstitial lung disease pathogenesis (including genetic factors causing familial disease) in children, and is expected to identify important preclinical markers of disease, pathways of disease regulation and novel potential targets for therapeutic intervention. For the future, there is a strong need for international collaboration that will allow collection of sufficiently large cohorts of patients with specific entities in order to perform proper therapeutic trials. As a prerequisite, however, a clear and standardised classification of the histopathology of the underlying conditions has to be developed. Such multicentre trials will help to reduce the still considerable morbidity and mortality in children with interstitial lung disease.

\section{REFERENCES}

1 Clement A, and committee members. Task force on chronic interstitial lung disease in immunocompetent children. Eur Respir J 2004; 24: 686-697.

2 American Thoracic Society/European Respiratory Society. International Multidisciplinary Consensus Classification of the Idiopathic Interstitial Pneumonias. Am J Respir Crit Care Med 2002; 165: 277-304.

3 Clement A, Henrion-Caude A, Fauroux B. The pathogenesis of interstitial lung diseases in children. Paediatr Respir Rev 2004; 5: 94-97.

4 Fan LL, Deterding RR, Langston C. Pediatric interstitial lung disease revisited. Pediatr Pulmonol 2004; 38: 369-378.

5 Gross TJ, Hunninghake GW. Idiopathic pulmonary fibrosis. N Engl J Med 2001; 345: 517-525.

6 Selman M, King TE, Pardo A. Idiopathic pulmonary fibrosis: prevailing and evolving hypotheses about its pathogenesis and implications for therapy. Ann Intern Med 2001; 134: 136-151.

7 Selman M, Pardo A. Idiopathic pulmonary fibrosis: an epithelial/fibroblastic cross-talk disorder. Respir Res 2002; 3: 3.
8 Selman M, Pardo A. The epithelial/fibroblastic pathway in the pathogenesis of idiopathic pulmonary fibrosis: tying loose ends. Am J Respir Cell Mol Biol 2003; 29: Suppl. 3, S93-S98.

9 Chua F, Gauldie J, Laurent GJ. Pulmonary fibrosis: searching for model answers. Am J Respir Cell Mol Biol 2005; 33: 9-13.

10 Chua F, Sly PD, Laurent GJ. Pediatric lung disease: from proteinases to pulmonary fibrosis. Pediatr Pulmonol 2005; 39: 392-401.

11 Noble PW, Homer RJ. Back to the future: historical perspective on the pathogenesis of idiopathic pulmonary fibrosis. Am J Respir Cell Mol Biol 2005; 33: 113-120.

12 Reynolds HY. Lung inflammation and fibrosis: an alveolar macrophage-centered perspective from the 1970s to 1980s. Am J Respir Crit Care Med 2005; 171: 98-102.

13 Reynolds HY, Gail DB, Kiley JP. Interstitial lung diseases where we started from and are now going. Sarcoidosis Vasc Diffuse Lung Dis 2005; 22: 5-12.

14 Kasper M, Haroske G. Alterations in the alveolar epithelium after injury leading to pulmonary fibrosis. Histol Histopathol 1996; 11: 463-483.

15 Barbas-Filho JV, Ferreira MA, Sesso A, Kairalla RA, Carvalho CR, Capelozzi VL. Evidence of type II pneumocyte apoptosis in the pathogenesis of idiopathic pulmonary fibrosis (IFP)/usual interstitial pneumonia (UIP). J Clin Pathol 2001; 54: 132-138.

16 Kinnula VL, Fattman CL, Tan RJ, Oury TD. Oxidative stress in pulmonary fibrosis: a possible role for redox modulatory therapy. Am J Respir Crit Care Med 2005; 172: 417-422.

17 Henson PM. Possible roles for apoptosis and apoptotic cell recognition in inflammation and fibrosis. Am J Respir Cell Mol Biol 2003; 29: Suppl. 3, S70-S76.

18 Khalil N, O'Connor R, Flanders K, Unruh H. TGF- $\beta 1$, but not TGF- $\beta 2$ or TGF- $\beta 3$ is differentially present in epithelial cells of advanced pulmonary fibrosis: an immunohistochemical study. Am J Respir Cell Mol Biol 1996; 14: 131-138.

19 Kuwano K, Hagimoto N, Tanaka T, et al. Expression of apoptosis-regulatory genes in epithelial cells in pulmonary fibrosis in mice. J Pathol 2000; 190: 221-229.

20 Kuwano K, Kunitake R, Maeyama T, et al. Attenuation of bleomycin-induced pneumopathy in mice by a caspase inhibitor. Am J Physiol Lung Cell Mol Physiol 2001; 280: L316-L325.

21 Kaminsli N. Microarray analysis of idiopathic pulmonary fibrosis. Am J Respir Cell Mol Biol 2003; 29: Suppl. 3, S32-S37.

22 Phan SH. Fibroblast phenotypes in pulmonary fibrosis. Am J Respir Cell Mol Biol 2003; 29: Suppl. 3, S87-S92.

23 Willis BC, Liebler JM, Luby-Phelps K, et al. Induction of epithelial-mesenchymal transition in alveolar epithelial cells by transforming growth factor- $\beta 1$ : potential role in idiopathic pulmonary fibrosis. Am J Pathol 2005; 166: 1321-1332.

24 Coker RK, Laurent GJ. Pulmonary fibrosis: cytokines in the balance. Eur Respir J 1998; 11: 1218-1221.

25 Millar A. Anti-cytokine therapy in fibrosing alveolitis: where are we now? Respir Res 2000; 1: 3-5.

26 Kasai H, Allen JT, Mason RM, Kamimura T, Zhang Z. TGF$\beta 1$ induces human alveolar epithelial to mesenchymal cell transition (EMT). Respir Res 2005; 6: 56. 
27 Massaro D, Massaro GD. Critical period for alveologenesis and early determinants of adult pulmonary disease. Am J Physiol Lung Cell Mol Physiol 2004; 287: L715-L717.

28 Orlandi A, Francesconi A, Marcellini M, Ferlosio A, Spagnoli LG. Role of ageing and coronary atherosclerosis in the development of cardiac fibrosis in the rabbit. Cardiovasc Res 2004; 64: 544-552.

29 Komi-Kuramochi A, Kawano M, Oda Y, et al. Expression of fibroblast growth factors and their receptors during fullthickness skin wound healing in young and aged mice. $J$ Endocrinol 2005; 186: 273-289.

30 Beggs ML, Nagarajan R, Taylor-Jones JM, Nolen G, Macnicol $M$, Peterson CA. Alterations in the TGF $\beta$ signaling pathway in myogenic progenitors with age. Aging Cell 2004; 3: 353-361.

31 Grutters JC, du Bois RM. Genetics of fibrosing lung diseases. Eur Respir J 2005; 25: 915-927.

32 Hartl D, Griese M. Interstitial lung disease in children genetic background and associated phenotypes. Respir Res 2005; 6: 32.

33 Cameron HS, Somaschini M, Carrera P, et al. A common mutation in the surfactant protein $C$ gene associated with lung disease. J Pediatr 2005; 146: 370-375.

34 Bullard JE, Wert SE, Whitsett JA, Dean M, Nogee LM. ABCA3 mutations associated with pediatric interstitial lung disease. Am J Respir Crit Care Med 2005; 172: 1026-1031.

35 Hilman BC. Diagnosis and treatment of ILD. Pediatr Pulmonol 1997; 23: 1-7.

36 Katzenstein AL, Myers JL. Idiopathic pulmonary fibrosis: clinical relevance of pathologic classification. Am J Respir Crit Care Med 1998; 157: 1301-1315.

37 Bush A. Paediatric interstitial lung disease: not just kid's stuff. Eur Respir J 2004; 24: 521-523.

38 King TE Jr. Clinical advances in the diagnosis and therapy of the interstitial lung diseases. Am J Respir Crit Care Med 2005; 172: 268-279.

39 Fan L, Langston C. Pediatric interstitial lung disease. Children are not small adults. Am J Respir Crit Care Med 2002; 165: 1466-1467.

40 Fan LL, Kozinetz CA, Deterding RR, Brugman SM. Evaluation of a diagnostic approach to pediatric interstitial lung disease. Pediatrics 1998; 101: 82-85.

41 Fan LL, Langston C. Chronic interstitial lung disease in children. Pediatr Pulmonol 1993; 16: 184-196.

42 Dinwiddie R, Sharief N, Crawford O. Idiopathic interstitial pneumonitis in children: a national survey in the United Kingdom and Ireland. Pediatr Pulmonol 2002; 34: 23-29.

43 Yeh TF, Lin YJ, Lin HC, et al. Outcomes at school age after postnatal dexamethasone therapy for lung disease of prematurity. N Engl J Med 2004; 350: 1304-1313.

44 Osika E, Muller MH, Boccon-Gibod L, et al. Idiopathic pulmonary fibrosis in infants. Pediatr Pulmonol 1997; 23: 49-54.

45 Desmarquest P, Tamalet A, Fauroux B, et al. Chronic interstitial lung disease in children: response to high-dose intravenous methylprednisolone pulses. Pediatr Pulmonol 1998; 26: 332-338.

46 Paul K, Klettke U, Moldenhauer J, et al. Increasing dose of methylprednisolone pulse therapy treats desquamative interstitial pneumonia in a child. Eur Respir J 1999; 14: 1429-1432.
47 Avital A, Godfrey S, Maayan C, Diamant Y, Springer C. Chloroquine treatment of interstitial lung disease in children. Pediatr Pulmonol 1994; 18: 356-360.

48 Balasubramanyan N, Murphy A, O'Sullivan J, O'Connell EJ. Familial interstitial lung diseases in children: response to chloroquine treatment in one sibling with desquamative interstitial pneumonitis. Pediatr Pulmonol 1997; 23: 55-61.

49 Block JA. Hydroxychloroquine and retinal safety. Lancet 1998; 351: 771.

50 Frosch M, Foell D. Wegener granulomatosis in childhood and adolescence. Eur J Pediatr 2004; 163: 425-434.

51 Lovell DJ, Ruth NM. Pediatric clinical research. Curr Opin Rheumatol 2005; 17: 265-270.

52 Pfleger A, Eber E, Popper H, Zach MS. Chronic interstitial lung disease due to Epstein-Barr virus infection in two infants. Eur Respir J 2000; 15: 803-806.

53 White CW, Wolf SJ, Korones DN, Sondheimer HM, Tosi MF, Yu A. Treatment of childhood angiomatous diseases with recombinant interferon alfa-2a. J Pediatr 1991; 118: 59-66.

54 De Blic J, Midulla F, Barbato A, et al. Bronchoalveolar lavage in children. ERS Task Force on bronchoalveolar lavage in children. European Respiratory Society. Eur Respir J 2000; 15: 217-231.

55 Seymour JF, Presneill JJ, Schoch OD, et al. Therapeutic efficacy of granulocyte-macrophage colony-stimulating factor in patients with idiopathic acquired alveolar proteinosis. Am J Respir Crit Care Med 2001; 163: 524-531.

56 Tazawa R, Hamano E, Arai T, et al. Granulocyte-macrophage colony-stimulating factor and lung immunity in pulmonary alveolar proteinosis. Am J Respir Crit Care Med 2005; 171: 1142-1149.

57 Price A, Manson D, Cutz E, Dell S. Pulmonary alveolar proteinosis associated with anti-GM-CSF antibodies in a child: successful treatment with inhaled GM-CSF. Pediatr Pulmonol 2006; 41: 367-370.

58 Moreno A, Maestre J, Balcells J, et al. Lung transplantation in young infants with interstitial pneumonia. Transplant Proc 2003; 35: 1951-1953.

59 Mallory GB, Spray TL. Paediatric lung transplantation. Eur Respir J 2004; 24: 839-845.

60 Kurland G, Michelson P. Bronchiolitis obliterans in children. Pediatr Pulmonol 2005; 39: 193-208.

61 Habib SB, Congleton J, Carr D, et al. Recurrence of recipient Langerhans' cell histiocytosis following bilateral lung transplantation. Thorax 1998; 53: 323-325.

62 Slebos DJ, Verschuuren EA, Koeter GH, et al. Bronchoalveolar lavage in a patient with recurrence of sarcoidosis after lung transplantation. J Heart Lung Transplant 2004; 3: 1010-1013.

63 Goldstein RH, Fine A. Potential therapeutic initiatives for fibrogenic lung diseases. Chest 1995; 108: 848-855.

64 Ziesche R, Hofbauer E, Wittmann K, Petkov V, Block LH. A preliminary study of long-term treatment with interferon $\gamma-1 b$ and low-dose prednisolone in patients with idiopathic pulmonary fibrosis. N Engl J Med 1999; 341: 1264-1269.

65 Raghu G, Brown KK, Bradford WZ, et al. A placebocontrolled trial of interferon $\gamma-1 \mathrm{~b}$ in patients with idiopathic pulmonary fibrosis. N Engl J Med 2004; 350: 125-133. 
66 Bajwa EK, Ayas NT, Schulzer M, Mak E, Ryu JH, Malhotra A. Interferon- $\gamma 1 \mathrm{~b}$ therapy in idiopathic pulmonary fibrosis. A metaanalysis. Chest 2005; 128: 203-206.

67 Raghu G, Johnson WC, Lockhart D, Mageto Y. Treatment of idiopathic pulmonary fibrosis with a new antifibrotic agent, pirfenidone: results of a prospective, open-label phase II study. Am J Respir Crit Care Med 1999; 159: 1061-1069.

68 Azuma A, Nukiwa T, Tsuboi E, et al. Double-blind, placebo-controlled trial of pirfenidone in patients with idiopathic pulmonary fibrosis.. Am J Respir Crit Care Med 2005; 171: 1040-1047.

69 Doty JD, Mazur JE, Judson MA. Treatment of sarcoidosis with infliximab. Chest 2005; 127: 1064-1071.

70 Tong Z, Dai H, Chen B, Abdoh Z, Guzman J, Costabel U. Inhibition of cytokine release from alveolar macrophages in pulmonary sarcoidosis by pentoxifylline. Chest 2003; 124: 1526-1532.
71 Zabel P, Entzian P, Dalhoff K, Schlaak M. Pentoxifylline in treatment of sarcoidosis. Am J Respir Crit Care Med 1997; 155: 1665-1669.

72 Utz JP, Limper AH, Kalra S, et al. Etanercept for the treatment of stage II and III progressive pulmonary sarcoidosis. Chest 2003; 124: 177-185.

73 Baughman RP, Judson MA, Teirstein AS, Moller DR, Lower EE. Thalidomide for chronic sarcoidosis. Chest 2002; 122: 227-232.

74 Sharief N, Crawford OF, Dinwiddie R. Fibrosing alveolitis and desquamative interstitial pneumonitis. Pediatr Pulmonol 1994; 17: 359-365.

75 Fan LL, Kozinetz CA. Factors influencing survival in children with chronic interstitial lung disease. Am J Respir Crit Care Med 1997; 156: 939-942.

76 Hacking D, Smyth R, Shaw N, Kokia G, Carty H, Heaf D. Idiopathic pulmonary fibrosis in infants: good prognosis with conservative management. Arch Dis Child 2000; 83: 152-157. 